\title{
Nuclear-magnetic-resonance quantum calculations of the Jones polynomial
}

\author{
Raimund Marx, ${ }^{1}$ Amr Fahmy, ${ }^{2, *}$ Louis Kauffman, ${ }^{3}$ Samuel Lomonaco, ${ }^{4}$ Andreas Spörl, ${ }^{1}$ Nikolas Pomplun, ${ }^{1}$ \\ Thomas Schulte-Herbrüggen, ${ }^{1}$ John M. Myers, ${ }^{5}$ and Steffen J. Glaser ${ }^{1}$ \\ ${ }^{1}$ Department of Chemistry, Technische Universität München, Lichtenbergstr. 4, D-85747 Garching, Germany \\ ${ }^{2}$ Biological Chemistry and Molecular Pharmacology, Harvard Medical School, 240 Longwood Avenue, Boston, Massachusetts 02115, USA \\ ${ }^{3}$ University of Illinois at Chicago, 851 S. Morgan Street, Chicago, Illinois 60607-7045, USA \\ ${ }^{4}$ University of Maryland Baltimore County, 1000 Hilltop Circle, Baltimore, Maryland 21250, USA \\ ${ }^{5}$ Cruft Laboratory, Harvard University, 19 Oxford Street, Cambridge, Massachusetts 02138, USA
}

(Received 10 September 2009; published 19 March 2010)

\begin{abstract}
The repertoire of problems theoretically solvable by a quantum computer recently expanded to include the approximate evaluation of knot invariants, specifically the Jones polynomial. The experimental implementation of this evaluation, however, involves many known experimental challenges. Here we present experimental results for a small-scale approximate evaluation of the Jones polynomial by nuclear magnetic resonance (NMR); in addition, we show how to escape from the limitations of NMR approaches that employ pseudopure states. Specifically, we use two spin-1/2 nuclei of natural abundance chloroform and apply a sequence of unitary transforms representing the trefoil knot, the figure-eight knot, and the Borromean rings. After measuring the nuclear spin state of the molecule in each case, we are able to estimate the value of the Jones polynomial for each of the knots.
\end{abstract}

DOI: 10.1103/PhysRevA.81.032319

PACS number(s): 03.67.Ac, 03.67.-a

\section{INTRODUCTION}

The Jones polynomial [1], a great discovery in knot theory, has recently become an interesting topic for quantum computing. In particular, the use of quantum computing has been discussed for approximately evaluating the Jones polynomial $V(z)$ at selected values of $z$. For a knot displayed as a braid of $n$ strands (specified in terms of a sequence of crossings), these are the values $z$ of the form $z=\exp (2 \pi i / k)$, where $k$ is an integer in the algorithm of Aharonov, Jones, and Landau (AJL) [2] (A description of the AJL algorithm can also be found in [3]). In Refs. [4,5] a quantum algorithm is given by Kauffman and Lomonaco (KL) for three-strand braids that can be used to evaluate the Jones polynomial at a continuous range of the argument of $z$. The approximate evaluation of the Jones polynomial by a quantum computer involves forming a product of unitary matrices and taking the trace of the product. The method of taking the trace described for the AJL algorithm and the KL algorithm requires that the quantum computer separately obtain an estimate of each of the diagonal elements of the unitary matrix; then these estimates are summed to yield an estimate of the trace. It is the taking of the trace that accounts for most of the computational cost of approximating the Jones polynomial.

The next section reviews the relation of the Jones polynomial for a braid to a unitary transformation composed of factors that correspond to braid crossings so the problem of evaluating the Jones polynomial reduces to the problem of evaluating the trace of a unitary matrix. An exposition of how the KL algorithm (which we use in this article) can be regarded as a special case of a generalization of the AJL algorithm is presented after that. In this sense this article and its sequels will be about experimental implementation of both the KL and the AJL quantum algorithms for computing the Jones polynomial.

\footnotetext{
*To whom correspondence should be addressed: amr_fahmy@ hms.harvard.edu
}

Following this, we present the method whereby an idealized nuclear magnetic resonance (NMR) quantum computer [6,7] can evaluate the trace of unitary matrix written as a product of factors all at once, that is, without having to evaluate diagonal elements of the unitary matrix separately. Experimental results for the evaluation of cases of the two-by-two matrix, and hence of the Jones polynomial for a braid of three strands, by use of NMR is the subject of the last section.

\section{THE JONES POLYNOMIAL AND UNITARY MATRICES}

The Jones polynomial [1] marked the beginning of a significant relationship between knot theory and statisical mechanics, particularly through the relationship of the polynomial with the Temperley-Lieb algebra, and through the explicit bracket state sum model [8-12]. From the topological side the Jones polynomial is striking because it can detect the difference between many knots and their mirror images. The general algorithm to find the Jones polynomial is in the $\sharp P$ complexity class, and so this is an algorithm worth understanding in the context of quantum computation.

Our approach to the Jones polynomnial in this article is based on representations of the Artin Braid Group [5]. The reader of this article can glean a quick introduction to ideas in knot theory and the braid group from Figs. 1, 2, and 3. A knot is an embedding of a circle in three-dimensional space, taken up to ambient isotopy. Two knots are said to be ambient isotopic if there is a time parameter family of embeddings starting with one knot and ending with the other one. A link is an embedding of several disjoint circles in three space, again taken up to ambient isotopy. It is convenient to use projection diagrams for knots and links to represent their topological type (up to ambient isotopy) and to record sufficient data for calculation and reconstruction. These diagrams are plane graphs with four edges incident to each node (4-regular plane graphs) and extra structure in the form of pictorial over and under crossing is given at each node. In Fig. 1 we illustrate the Reidemeister moves for such diagrams. 
I

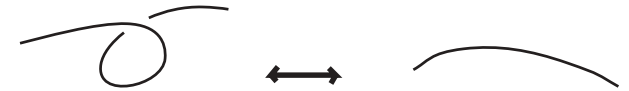

II

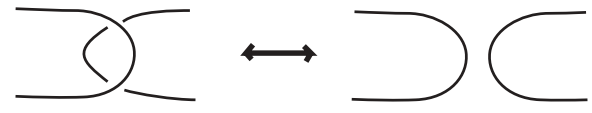

III

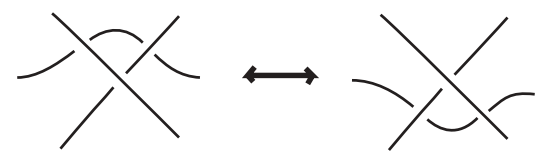

FIG. 1. Reidemeister moves.

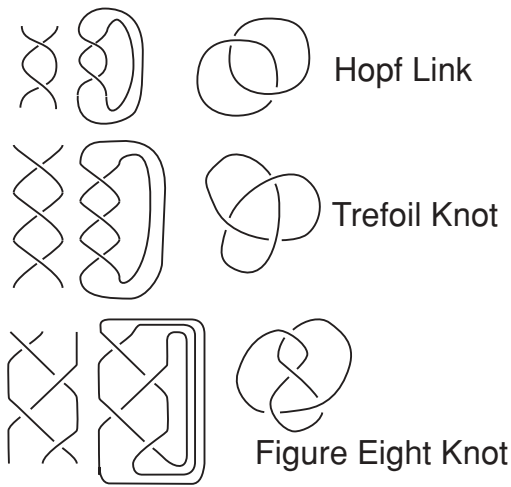

FIG. 3. Braid closures.
These moves accomplish ambient isotopies of the embeddings that correspond to given diagrams, and it is a fact that two knot or link diagrams represent ambient isotopic configurations in three-dimensional space if and only if the two diagrams can be transformed one to another by a sequence of Reidemeister moves. A braid in $B_{n}$ is a collection of arcs in three-space, starting at a selected set of $n$ points and embedded in such a way that the strands move always downward with respect to a choice of vertical direction, until they reach a second copy of this set of points. In the diagrams of Figs. 2 and 3 , the reader will see braids drawn so that the vertical direction corresponds to the direction up and down the page on which the diagrams are drawn. Braids are taken up to isotopy in three dimensions that fixes their end points and does not disturb the levels of the points on any given braid strand. Braids can be multiplied by attaching the bottom end points of one braid to the top end points of another braid. If $b$ and $b^{\prime}$ are braids of $n$ strands, we denote their product by $b b^{\prime}$.

In Fig. 2, we illustrate the generators $\sigma_{1}, \sigma_{2}, \sigma_{3}$ of the four-strand braid group $B_{4}$. With $n$ strands there are $n-1$ generators $\sigma_{1}, \sigma_{2}, \cdots \sigma_{n-1}$, where $\sigma_{i}$ represents a right-handed twist of strands $i$ and $i+1$, with all other strands proceeding straight down with no twist. Each $\sigma_{i}$ is undone by multiplication by $\sigma_{i}^{-1}$, a left-handed twist of the same two strands. The result is that the braid group $B_{n}$ lives up to its name and forms a group under the multiplication that we have described. The identity element in the group, denoted by $\mathbf{1}$ or by $\mathbf{1}_{n}$, consists in $n$ descending straight strands with no twist. In Fig. 2 we have illustrated these inverses, and we have illustrated the identities $\sigma_{1} \sigma_{2} \sigma_{1}=\sigma_{2} \sigma_{1} \sigma_{2}$ and $\sigma_{1} \sigma_{3}=\sigma_{3} \sigma_{1}$. In

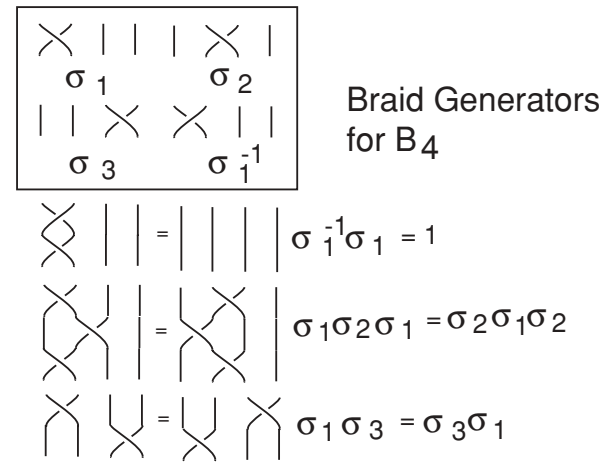

FIG. 2. Braid generators and relations. general, in $B_{n}$ a complete set of relations for the group consists in $\sigma_{i} \sigma_{i+1} \sigma_{i}=\sigma_{i+1} \sigma_{i} \sigma_{i+1}$ and $\sigma_{i} \sigma_{j}=\sigma_{j} \sigma_{i}$ when $|i-j|>1$. In this article we will use only the three-strand braid group, with its two generators $\sigma_{1}$ and $\sigma_{2}$.

In Fig. 3 we illustrate how knots and links can be obtained by taking closures (attach the top strand points to the bottom strand points) of braids. In fact, by a classical theorem of J. W. Alexander, every knot and link can be obtained as the closure of a braid (always in more than one way). In Fig. 3 we see the Hopf link, the trefoil knot, and the figure-eight knot as braid closures.

The key idea behind the present quantum algorithms to compute the Jones polynomial is to use unitary representations of the braid group derived from Temperley-Lieb algebra representations that take the form

$$
\rho\left(\sigma_{i}\right)=A \mathbf{1}+A^{-1} U_{i}
$$

where $\sigma_{i}$ is a standard generator of the Artin braid group, $A$ is a complex number of unit length, $\mathbf{1}$ is the two-by-two unit matrix, and $U_{i}$ is a symmetric real matrix that is part of a representation of the Temperley-Lieb algebra. For more details about this strategy and the background information about the Jones polynomial, the bracket model for the Jones polynomial and the Temperley-Lieb algebra the reader may wish to consult Refs. [1,2,4,5,8-15]. In the following mathematical description, we have given a minimal exposition of the structure of such representations. As an example, the reader may wish to examine Fig. 3 and note that the figure-eight knot is the closure of the braid $\sigma_{1} \sigma_{2}^{-1} \sigma_{1} \sigma_{2}^{-1}$. In the next sections we describe the unitary representations of the braid group in more detail, and one can follow these with the specific example of the figure-eight knot. Indeed, we have done this all the way through in our experiments with NMR quantum computing.

\section{A. Two projectors and a unitary representation of the three-strand braid group}

It is useful to think of the Temperley-Lieb algebra as generated by projections $e_{i}=U_{i} / \delta$ so that $e_{i}^{2}=e_{i}$ and $e_{i} e_{i \pm 1} e_{i}=$ $\tau e_{i}$, where $\tau=\delta^{-2}$ and $e_{i}$ and $e_{j}$ commute for $|i-j|>1$. With this in mind, consider elementary projectors $e=|A\rangle\langle A|$ and $f=|B\rangle\langle B|$. We assume that $\langle A \mid A\rangle=\langle B \mid B\rangle=1$ so that $e^{2}=e$ and $f^{2}=f$. Now note that

$$
\text { efe }=|A\rangle\langle A \mid B\rangle\langle B \mid A\rangle\langle A|=\langle A \mid B\rangle\langle B \mid A\rangle e=\tau e .
$$


Thus

$$
e f e=\tau e,
$$

where $\tau=\langle A \mid B\rangle\langle B \mid A\rangle$.

This algebra of two projectors is the simplest instance of a representation of the Temperley-Lieb algebra. In particular, this means that a representation of the three-strand braid group is naturally associated with the algebra of two projectors.

Quite specifically if we let $\langle A|=(a, b)$ and $|A\rangle=(a, b)^{T}$ the transpose of this row vector, then

$$
e=|A\rangle\langle A|=\left[\begin{array}{ll}
a^{2} & a b \\
a b & b^{2}
\end{array}\right]
$$

is a standard projector matrix when $a^{2}+b^{2}=1$. To obtain a specific representation, let $e_{1}=\left[\begin{array}{ll}1 & 0 \\ 0 & 0\end{array}\right]$ and $e_{2}=\left[\begin{array}{cc}a^{2} & a b \\ a b & b^{2}\end{array}\right]$. It is easy to check that $e_{1} e_{2} e_{1}=a^{2} e_{1}$ and that $e_{2} e_{1} e_{2}=a^{2} e_{2}$. Note also that $e_{1} e_{2}=\left[\begin{array}{cc}a^{2} & a b \\ 0 & 0\end{array}\right]$ and $e_{2} e_{1}=\left[\begin{array}{cc}a^{2} & 0 \\ a b & 0\end{array}\right]$. We define

$$
U_{i}=\delta e_{i}
$$

for $i=1,2$ with $a^{2}=\delta^{-2}$. Then we have, for $i=1,2$

$$
U_{i}^{2}=\delta U_{i}, \quad U_{1} U_{2} U_{1}=U_{1}, \quad U_{2} U_{1} U_{2}=U_{2} .
$$

Thus we have a representation of the Temperley-Lieb algebra on three strands. See Ref. [11] for a discussion of the properties of the Temperley-Lieb algebra.

Note also that we have

$$
\operatorname{trace}\left(U_{1}\right)=\operatorname{trace}\left(U_{2}\right)=\delta,
$$

while

$$
\operatorname{trace}\left(U_{1} U_{2}\right)=\operatorname{trace}\left(U_{2} U_{1}\right)=1,
$$

where trace denotes the usual matrix trace. We will use these results on the traces of these matrices in the next section. Now we return to the matrix parameters: Since $a^{2}+b^{2}=1$ this means that $\delta^{-2}+b^{2}=1$ whence $b^{2}=1-\delta^{-2}$. Therefore $b$ is real when $\delta^{2}$ is greater than or equal to 1 .

We are interested in the case where $\delta=-A^{2}-A^{-2}$ and $A$ is a unit complex number. Under these circumstances the braid group representation

$$
\rho\left(\sigma_{i}\right)=A \mathbf{1}+A^{-1} U_{i}
$$

will be unitary whenever $U_{i}$ is a real symmetric matrix. Thus we will obtain a unitary representation of the three-strand braid group $B_{3}$ when $\delta^{2} \geqslant 1$.

For any $A$ with $d=-A^{2}-A^{-2}$ these formulas define a representation of the braid group. With $A=\exp (i \theta)$, we have $d=-2 \cos (2 \theta)$. We find a specific range of angles $\theta$ in the following disjoint union of angular intervals

$$
\begin{gathered}
\theta \in[0, \pi / 6] \sqcup[\pi / 3,2 \pi / 3] \sqcup[5 \pi / 6,7 \pi / 6] \\
\sqcup[4 \pi / 3,5 \pi / 3] \sqcup[11 \pi / 6,2 \pi]
\end{gathered}
$$

that give unitary representations of the three-strand braid group. Thus a specialization of a more general representation of the braid group gives rise to a continuous family of unitary representations of the braid group.

\section{B. A Quantum algorithm for the Jones polynomial on three-strand braids}

We gave above an example of a unitary representation of the three-strand braid group. In fact, we can use this representation to compute the Jones polynomial for closures of three-braids, and therefore this representation provides a test case for the corresponding quantum computation. We now analyze this case by first making explicit how the bracket polynomial is computed from this representation. This unitary representation and its application to a quantum algorithm first appeared in Ref. [4]. When coupled with the Hadamard test, this algorithm gets values for the Jones polynomial in polynomial time in the same way as the AJL algorithm [2]. It remains to be seen how fast these algorithms are in principle when asked to compute the polynomial itself rather than certain specializations of it.

First recall that the representation depends on two matrices $U_{1}$ and $U_{2}$ with

$$
U_{1}=\left[\begin{array}{ll}
\delta & 0 \\
0 & 0
\end{array}\right] \text { and } U_{2}=\left[\begin{array}{cc}
\delta^{-1} & \sqrt{1-\delta^{-2}} \\
\sqrt{1-\delta^{-2}} & \delta-\delta^{-1}
\end{array}\right]
$$

The representation is given on the two braid generators by

$$
\rho\left(\sigma_{1}\right)=A \mathbf{1}+A^{-1} U_{1}
$$

and

$$
\rho\left(\sigma_{2}\right)=A \mathbf{1}+A^{-1} U_{2}
$$

for any $A$ with $\delta=-A^{2}-A^{-2}$ and with $A=\exp (i \theta)$, then $\delta=-2 \cos (2 \theta)$. We get the specific range of angles $\theta \in[0, \pi / 6] \sqcup[\pi / 3,2 \pi / 3] \sqcup[5 \pi / 6,7 \pi / 6] \sqcup[4 \pi / 3$, $5 \pi / 3] \sqcup[11 \pi / 6,2 \pi]$ that give unitary representations of the three-strand braid group.

Note that $\operatorname{tr}\left(U_{1}\right)=\operatorname{tr}\left(U_{2}\right)=\delta$ while $\operatorname{tr}\left(U_{1} U_{2}\right)=$ $\operatorname{tr}\left(U_{2} U_{1}\right)=1$. If $b$ is any braid, let $I(b)$ denote the sum of the exponents in the braid word that expresses $b$. For $b$ a three-strand braid, it follows that

$$
\rho(b)=A^{I(b)} \mathbf{1}+\tau(b),
$$

where $\tau(b)$ is a sum of products in the Temperley-Lieb algebra involving $U_{1}$ and $U_{2}$. Since the Temperley-Lieb algebra in this dimension is generated by $\mathbf{1}, U_{1}, U_{2}, U_{1} U_{2}$, and $U_{2} U_{1}$, it follows that

$$
\langle\bar{b}\rangle=A^{I(b)} \delta^{2}+\operatorname{tr}[\tau(b)],
$$

where $\bar{b}$ denotes the standard braid closure of $b$ and the sharp brackets denote the bracket polynomial. From this we see at once that

$$
\langle\bar{b}\rangle=\operatorname{tr}[\rho(b)]+A^{I(b)}\left(\delta^{2}-2\right) .
$$

It follows from this calculation that the question of computing the bracket polynomial for the closure of the three-strand braid $b$ is mathematically equivalent to the problem of computing the trace of the matrix $\rho(b)$.

The matrix in question is a product of unitary matrices, the quantum gates that we have associated with the braids $\sigma_{1}$ and $\sigma_{2}$. The entries of the matrix $\rho(b)$ are the results of preparation and detection for the two-dimensional basis of qubits for our machine:

$$
\langle i|\rho(b)| j\rangle \text {. }
$$


Given that the computer is prepared in $|j\rangle$, the probability of observing it in state $|i\rangle$ is equal to $|\langle i|\rho(b)| j\rangle|^{2}$. Thus we can, by running the quantum computation repeatedly, estimate the absolute squares of the entries of the matrix $\rho(b)$. This will not yield the complex phase information that is needed for either the trace of the matrix or the absolute value of that trace.

However, we do know how to write a quantum algorithm to compute the trace of a unitary matrix (via the Hadamard test). Since $\rho(b)$ is unitary, we can use this approach to approximate the trace of $\rho(b)$. This yields a quantum algorithim for the Jones polynomial for three-stand braids (evaluated at points $A$ such that the representation is unitary). Knowing $\operatorname{tr}[\rho(b)]$ from the quantum computation, we then have the formula for the bracket, as above,

$$
\langle\bar{b}\rangle=\operatorname{trace}[\rho(b)]+A^{I(b)}\left(\delta^{2}-2\right) .
$$

Then the normalized polynomial, invariant under all three Reidemeister moves is given by

$$
f(\bar{b})=\left(-A^{3}\right)^{-I(b)}\langle\bar{b}\rangle .
$$

Finally, the Jones polynomial in its usual form is given by the formula

$$
V(\bar{b})(t)=f(\bar{b})\left(t^{-1 / 4}\right) .
$$

Thus we conclude that our quantum computer can approximate values of the Jones polynomial.

\section{RELATIONSHIP WITH THE AJL ALGORITHM}

Here is how the KL algorithm described in the previous section becomes a special case of a generalization of the AJL algorithm: Here we use notation from the AJL article. In that article, the generators $U_{i}$ (in our previous notation) for the Temperley-Lieb algebra, are denoted by $E_{i}$.

Let $L_{k}=\lambda_{k}=\sin (k \theta)$. For the time being $\theta$ is an arbitrary angle. Let $A=i \exp (i \theta / 2)$ so that $\delta=-A^{2}-A^{-2}=2 \cos (\theta)$.

We need to choose $\theta$ so that $\sin (k \theta)$ is non-negative for the range of $k$ 's we use (these depend on the choice of line graph as in AJL). And we insist that $\sin (k \theta)$ is nonzero except for $k=0$. Then it follows from trigonometry that $\left(L_{k-1}+L_{k+1}\right) / L_{k}=\delta$ for all $k$.

Recall that the representation of the Temperley-Lieb algebra in AJL is given in terms of $E_{i}$ such that $E_{i}^{2}=\delta E_{i}$ and the $E_{i}$ satisfy the Temperley-Lieb relations. Each $E_{i}$ acts nontrivially at the $i$ and $i+1$ places in the bit-string basis for the space and each $E_{i}$ is based on $L_{a-1}, L_{a}, L_{a+1}$, where $a=z(i)$ is the end point of a walk described by the bitstring using only first $(i-1)$ bits. Bitstrings represent walks on a line graph. Thus 1011 represents the walk Right, Left, Right, Right ending at node number 3 in

$$
1-----2-----3-----4 \text {. }
$$

For $\quad p=1011, \quad z(1)=1, z(2)=2, z(3)=1, z(4)=2$, $z(5)=3$.

More precisely, if we let

$$
|v(a)\rangle=\left[\sqrt{L_{a-1} / L_{a}}, \sqrt{L_{a+1} / L_{a}}\right]^{T}
$$

(i.e., this is a column vector; $T$ denotes transpose), then

$$
E_{i}=|v(z(i))\rangle\langle v(z(i))| .
$$

Here it is understood that this refers to the action on the bitstrings

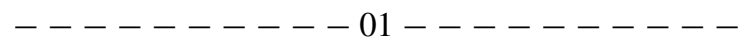

and

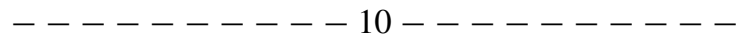

obtained from the given bitstring by modifying the $i$ and $i+1$ places. The basis order is 01 before 10 . Conceptually, this is a useful description, but it also helps to have the specific formulas laid out.

Now look at the special case of a line graph with three nodes and two edges:

$$
1-----2----3 .
$$

The only admissible binary sequences are $|110\rangle$ and $|101\rangle$, so the space corresponding to this graph is two dimensional, and it is acted on by $E_{1}$ with $z(1)=1$ in both cases (the empty walk terminates in the first node) and $E_{2}$ with $z(2)=2$ for $|110\rangle$ and $z(2)=2$ for $|101\rangle$. Then we have

$$
\begin{gathered}
E_{1}|110\rangle=0, E_{1}|101\rangle=\delta|101\rangle, \\
E_{2}|x y z\rangle=|v\rangle\langle v \mid x y z\rangle
\end{gathered}
$$

$($ xyz $=101$ or 110$)$, where $v=(\sqrt{1 / \delta}, \sqrt{\delta-1 / \delta})^{T}$.

If one compares this two-dimensional representation of the three strand Temperley-Lieb algebra and the corresponding braid group representation, with the representation Kauffman and Lomonaco use in their article, it is clear that it is the same [up to the convenient replacement of $A=\exp (i \theta)$ by $A=i \exp (i \theta / 2)]$. The trace formula of AJL is a variation of the trace formula that Kauffman and Lomonaco use. Note that the AJL algorithm as formulated in [2] does not use the continuous range of angles that are available to the $\mathrm{KL}$ algorithm. In the sequel to this article and in a separate article on the mathematics, we shall show how the entire AJL algorithm generalizes to continuous angular ranges.

\section{THEORY OF AN NMR SPECTROMETER USED AS A QUANTUM COMPUTER}

By convention, a quantum computer as conceived in theory is assumed to yield an outcome associated with a quantum measurement of some (possibly mixed) quantum state. In contrast, NMR machines implement a restricted version of an expectation-value quantum computer (EVQC), which in place of an outcome yields, to some finite precision, the expectation value for a measurement of a (again, possibly mixed) quantum state [7]. Reflecting facts of NMR spectrometers, an NMR quantum computer (NMRQC) implements only the special measurement operators discussed in Ref. [17], and these measurement operators all have zero trace.

Here are the details. For a Hermitian measurement operator $M$ applied to a density matrix $\rho$, the EVQC of precision $\epsilon$ yields a complex number $z$ such that

$$
|z-\operatorname{tr}(M \rho)| \leqslant \epsilon \Lambda(M),
$$

where $\Lambda(M)$ is the difference between the minimum and the maximum eigenvalue of the measurement operator $M$, which 
is just the possible range to the trace as $\rho$ varies over all possible density matrices. [The factor $\Lambda(M)$ makes limitations of resolution immune to the mere analytic trick of multiplying the measurement operator by a constant.]

The measurement operators of main interest for the algorithm by which we estimate the trace of a unitary operator are $I_{1 x}$ and $I_{1 y}$, shortly to be defined.

\section{A. Thermal equilibrium and initial state preparation}

To first order, the initial thermal state density operator of an ensemble (very large number) of quantum systems with $n+1$ qubits each [18] is given by

$$
\rho_{t h} \approx \frac{1}{N}\left(\mathbf{1}_{\mathbf{n}+\mathbf{1}}-\sum_{l=1}^{n+1} \alpha_{l} I_{l z}\right)
$$

with $\alpha_{l}=\frac{\hbar \omega_{l}}{k T}, \mathbf{1}_{\mathbf{n}+\mathbf{1}}$ is the $2^{n+1}$-by- $2^{n+1}$ unit matrix

$$
I_{l z}=\frac{1}{2} \mathbf{1} \otimes \cdots \otimes \mathbf{1} \otimes \sigma_{z} \otimes \mathbf{1} \otimes \cdots \otimes \mathbf{1},
$$

where the Pauli matrix $\sigma_{z}$ appears as the $l$ th term in the product, $\omega_{l}$ is the resonance frequency of qubit $l, k$ is Boltzmann's constant, $T$ is temperature, and $N=2^{n+1}$.

The initial density operator required for our algorithm is given by

$$
\rho_{0}=\frac{1}{N}\left(\mathbf{1}_{\mathbf{n}+\mathbf{1}}-\alpha_{1} I_{1 z}\right),
$$

which can be prepared from $\rho_{t h}$ by a variety of methods [19].

\section{B. Algorithm to estimate the trace of $U$}

The method presented here is based on the algorithm that first appeared in Ref. [20]. As mentioned above we assume that $U$ is given in the form of local operations on $n$ qubits. Given a program for $U$, Barenco et al. [21] describe a procedure to construct a program or local operations for the operator controlled-U, $c U . c U$ operates on $n+1$ qubits, does not affect the first qubit, applies $U$ on the remaining $n$ qubits if the first qubit is $|1\rangle$ and does nothing otherwise:

$$
\begin{gathered}
c U|1\rangle|\psi\rangle=(\mathbf{1} \otimes U)|1\rangle|\psi\rangle=|1\rangle U|\psi\rangle \\
c U|0\rangle|\psi\rangle=|0\rangle|\psi\rangle .
\end{gathered}
$$

In block matrix form, $c U$ is given by:

$$
c U=\left(\begin{array}{cc}
\mathbf{1}_{\mathbf{n}} & 0 \\
0 & U
\end{array}\right) .
$$

We now describe our algorithm:

Step 1: Prepare the density operator:

$$
\rho_{1}=\frac{1}{N}\left(\mathbf{1}_{\mathbf{n}+\mathbf{1}}-\alpha_{1} I_{1 x}\right)=\frac{1}{N} \mathbf{1}_{\mathbf{n}+\mathbf{1}}-\frac{\alpha_{1}}{2 N}\left(\begin{array}{cc}
0 & \mathbf{1}_{\mathbf{n}} \\
\mathbf{1}_{\mathbf{n}} & 0
\end{array}\right),
$$

where

$$
I_{1 x}=\frac{1}{2} \sigma_{x} \otimes \mathbf{1}_{\mathbf{n}} \quad \text { and } \quad \sigma_{x}=\left[\begin{array}{ll}
0 & 1 \\
1 & 0
\end{array}\right] .
$$

Step 2: Apply $c U$ to $\rho_{1}$ :

$$
\rho_{2}=c U \rho_{1} c U^{\dagger}=\frac{1}{N} \mathbf{1}_{\mathbf{n}+\mathbf{1}}-\frac{\alpha_{1}}{2 N}\left(\begin{array}{cc}
0 & U^{\dagger} \\
U & 0
\end{array}\right) .
$$

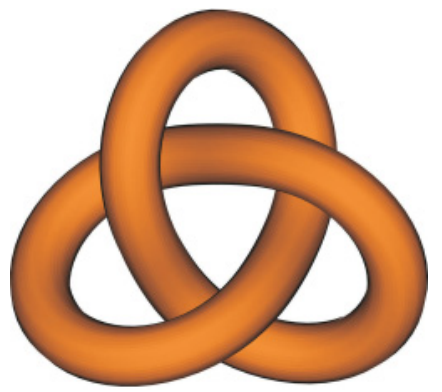

FIG. 4. (Color online) The trefoil knot generated by the sequence $\sigma_{1}^{3}$.

Step 3: Measure $\left\langle I_{1 x}+i I_{1 y}\right\rangle$ to estimate

$$
\operatorname{trace}\left(\left(I_{1 x}+i I_{1 y}\right) \rho_{2}\right)=\frac{\alpha_{1}}{N} \operatorname{trace}(U)
$$

where

$$
I_{1 y}=\frac{1}{2} \sigma_{y} \otimes \mathbf{1}_{\mathbf{n}} \text { and } \sigma_{y}=\left[\begin{array}{rr}
0 & -i \\
i & 0
\end{array}\right] .
$$

By Eq. (3) the result of this measurement is a complex number, $\mathrm{z}$, such that

$$
\begin{aligned}
& \left|\operatorname{Re}(z)-\frac{\alpha_{1}}{N} \operatorname{Re}[\operatorname{trace}(U)]\right| \leqslant \epsilon \\
& \left|\operatorname{Im}(z)-\frac{\alpha_{1}}{N} \operatorname{Im}[\operatorname{trace}(U)]\right| \leqslant \epsilon .
\end{aligned}
$$

It follows that the measurement result satisfies

$$
|z-\operatorname{trace}(U)| \leqslant \sqrt{2} N \epsilon / \alpha_{1} .
$$

\section{EXAMPLE KNOTS AND EXPERIMENTAL RESULTS}

Experimental results for two knots and one link on three strands were obtained using the methods outlined above, see Figs. 4 to 9 . Specifically, we present results for the trefoil knot, the figure-eight knot, the Borromean rings. A two-spin system (details of the molecule and pulse sequences are given later) was used, the initial state given by the

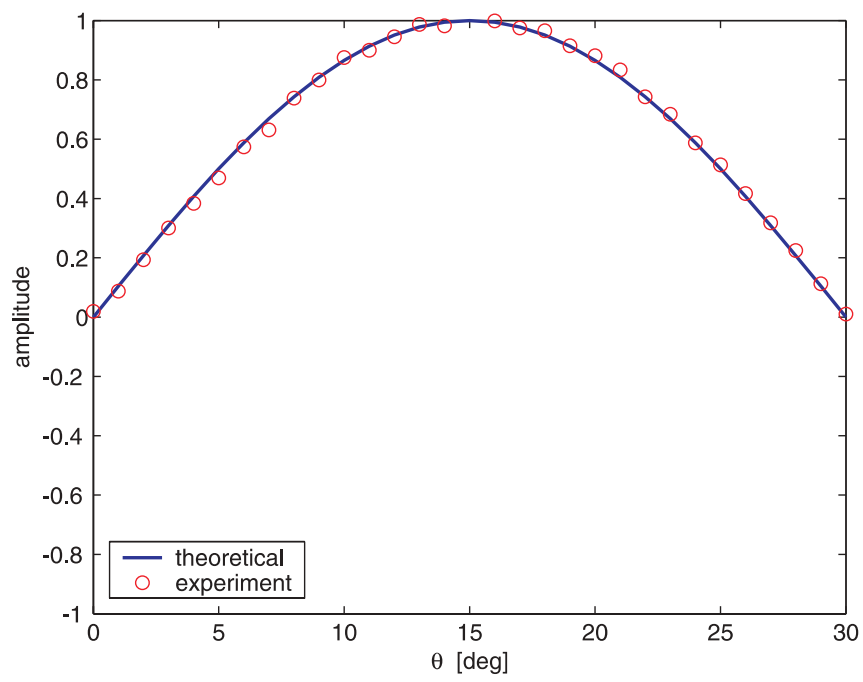

FIG. 5. (Color online) Experimental results for the trefoil knot. 


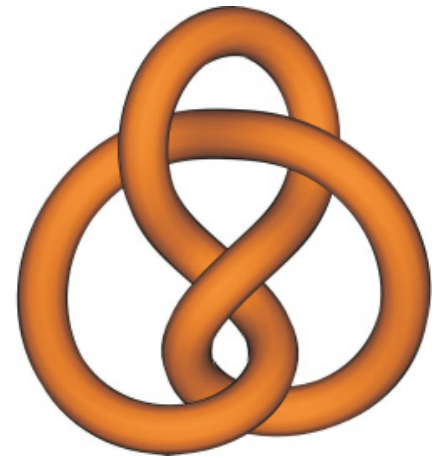

FIG. 6. (Color online) The figure-eight knot generated by the sequence $\sigma_{1} \sigma_{2}^{-1} \sigma_{1} \sigma_{2}^{-1}$.

density operator proportional to $I_{1 x}$ was prepared and a reference spectrum was then collected. This was followed by application of a controlled-unitary operator corresponding to and representing each knot separately found from the representation

$$
s_{1}=\rho\left(\sigma_{1}\right) \text { and } s_{2}=\rho\left(\sigma_{2}\right) .
$$

Measurement of the expectation value of $I_{1 x}+i I_{1 y}$ after applying the controlled-unitary operator yields the trace of the unitary operator representing the knot and thus the estimate of the Jones polynomial for each knot.

As an example, to estimate the Jones polynomial for the figure-eight knot, which is the closure of the braid $\sigma_{1} \sigma_{2}^{-1} \sigma_{1} \sigma_{2}^{-1}$, we will apply the sequence of unitary transforms $c s_{1} \quad c s_{2}^{-1} \quad c s_{1} c s_{2}^{-1}$ and then measure the expectation value of $I_{1 x}+i I_{1 y}$, where $c s$ is controlled-s.

For each of the three knots, the Jones polynomial was estimated at the complex numbers $e^{i \theta}$ for all $\theta$ in the range $0 \leqslant$ $\theta \leqslant \pi / 6$ at single degree increments (31 values). Comparison to the theoretical values shows excellent correspondence with experimental observations. Furthermore, the Jones polynomial itself for each of these knots can be constructed from the experimental results.

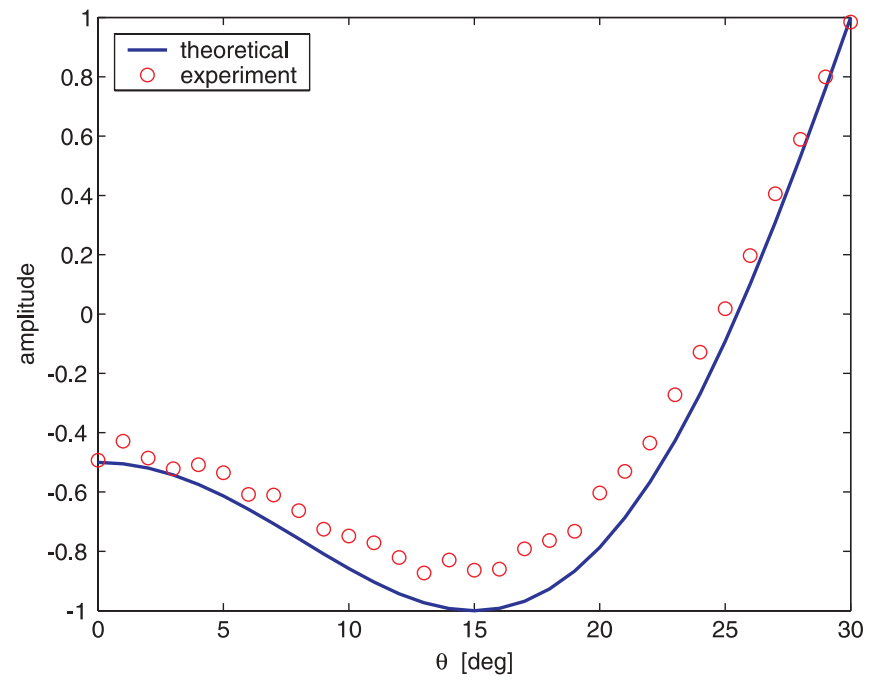

FIG. 7. (Color online) Experimental results for the figure-eight knot.

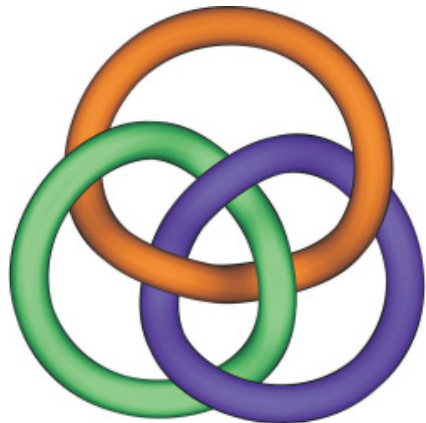

FIG. 8. (Color online) The Borromean rings generated by the sequence $\sigma_{1} \sigma_{2}^{-1} \sigma_{1} \sigma_{2}^{-1} \sigma_{1} \sigma_{2}^{-1}$.

\section{A. Experimental setup and molecule}

The experimental implementation of the algorithm to estimate the trace of the unitary operator involves finding a suitable molecule (here: ${ }^{13} \mathrm{C}$-chloroform) that can be used as the hardware of the NMR quantum computer, designing a pulse sequences that implement the desired unitary transformation with high fidelity and adjusting and calibrating the experimental setup. This involves adjusting the homogeneity of the magnet, adjusting the radiofrequency pulse lengths, power and irradiation frequencies and cancellation of all signals that originate from ${ }^{12} \mathrm{C}$-chloroform, decoupling of ${ }^{1} \mathrm{H}$ during ${ }^{13} \mathrm{C}$ detection, separate detection of $I_{x}$ and $I_{y}$.

The precision of NMR measurements is very high. But the accuracy of the measured value may be decreased by several experimental imperfections, e.g., inhomogeneity and miscalibration of radiofrequency pulses, relaxation effects, and so on. Such experimental imperfections are the reason why our experimental results do not precisely match the theoretical expectations.

All experiments were performed on a Bruker Avance DMX 750 NMR spectrometer, equipped with a TXI 5-mm probe head with XYZ gradients. The sample was a 9:1 mixture of chloroform and deuterated acetone. It naturally contained about $1 \%$ of ${ }^{13} \mathrm{C}-{ }^{1} \mathrm{H}$ chloroform which was the

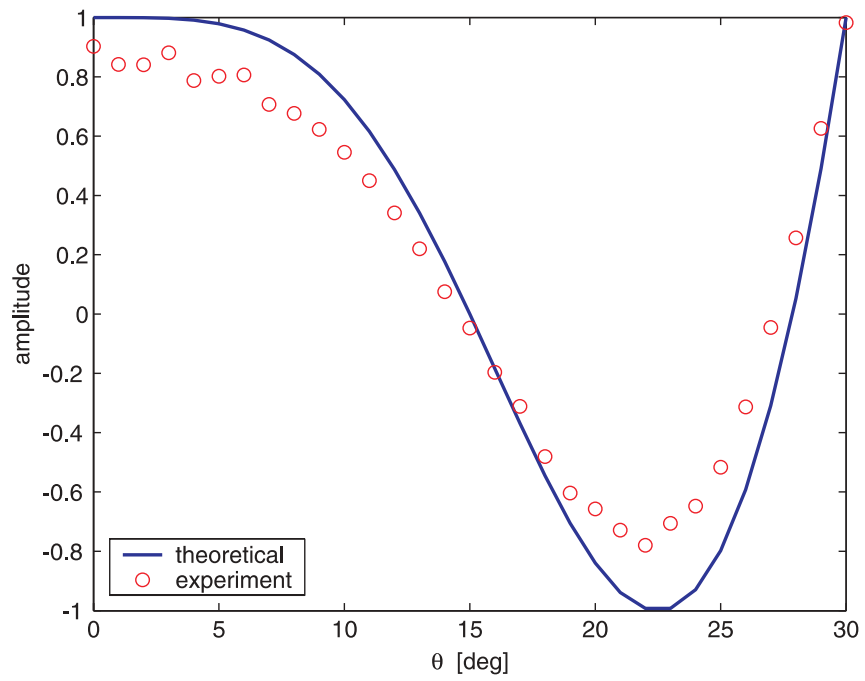

FIG. 9. (Color online) Experimental results for the Borromean rings. 

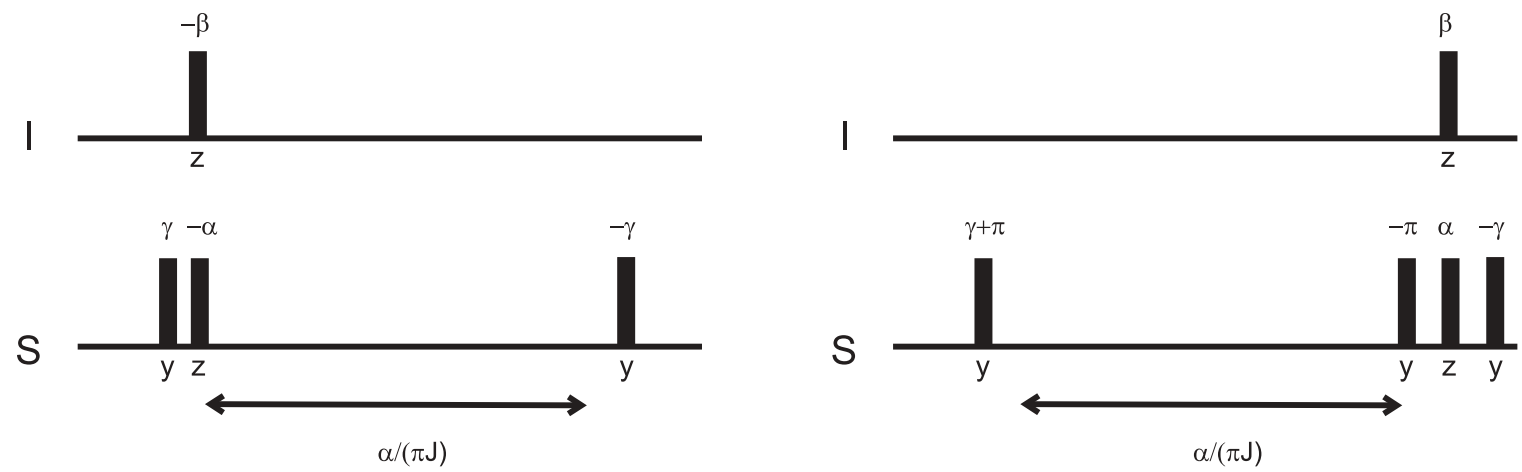

FIG. 10. Pulse sequence to implement a controlled- $s_{1,2}$ operation (left) and its inverse (right). For $s_{1}$ set $\gamma$ to 0 . (To obtain a propagator of $\mathbf{1} \oplus s_{1,2}$ respectively its inverse, we have to apply a global phase factor of $e^{\mp i \pi \beta / 2}$. The propagator of a pulse on the second spin- $1 / 2$ is defined as $U_{\text {pulse }}:=e^{-i \epsilon S_{v}}$, with $S_{v}:=\left(1 \otimes \sigma_{v} / 2\right), \epsilon \in\{\alpha, \beta, \gamma\}$ and $v \in\{y, z\}$, where $\alpha=0.5 \pi-2 \theta, \beta=0.5 \pi+\theta$ and $\gamma=\tan ^{-1}\left[\cos 4 \theta / \sqrt{4 \cos ^{2} 2 \theta-1}\right]+\pi / 2$ and $0 \leqslant \theta \leqslant \pi / 6$. For the free evolution $U_{\text {evolution }}:=e^{-i \pi t J 2 I_{z} S_{z}}$ with $2 I_{z} S_{z}:=2\left(\sigma_{z} / 2 \otimes \sigma_{z} / 2\right)$. The pulse sequence is applied to the initial density operator $I_{1 x}$.

active compound that represented the hardware of our NMR quantum computer. The spin systems Larmor frequencies were 188.6349005 MHz for ${ }^{13} \mathrm{C}$ and $750.1354275 \mathrm{MHz}$ for ${ }^{1} \mathrm{H}$. The corresponding chemical shifts are 77.2 and $7.235 \mathrm{ppm}$, respectively. The two spin- $1 / 2$ nuclei of ${ }^{13} \mathrm{C}-{ }^{1} \mathrm{H}$ chloroform interact through scalar coupling. The corresponding coupling constant is $J=209.5 \mathrm{~Hz}$. The longitudinal relaxation times $\left(T_{1}\right)$ and transversal relaxation times $\left(T_{2}\right)$ of both spin-1/2 nuclei are: ${ }^{13} \mathrm{C} T_{1}: 21.8 \mathrm{~s},{ }^{13} \mathrm{C} T_{2}: 0.19 \mathrm{~s},{ }^{1} \mathrm{H} T_{1}: 6.1 \mathrm{~s},{ }^{1} \mathrm{H}$ $T_{2}$ : $0.48 \mathrm{~s}$. In order to suppress the signal of $99 \%{ }^{12} \mathrm{C}-{ }^{1} \mathrm{H}$ chloroform and to prepare the initial operator $I_{x}$, where $I$ corresponds to ${ }^{13} \mathrm{C}\left({ }^{1} \mathrm{H}\right.$ will be referred to as $S$ ), the following preparation sequence was used in all experiments: the ${ }^{1} \mathrm{H}$ spins were saturated by cw irradiation. Subsequently they were dephased by applying a $9.9 \mu \mathrm{s} 90^{\circ}\left({ }^{1} \mathrm{H}\right)$ pulse followed by a $B_{0}$ gradient. This sequence of $90^{\circ}$ pulse and gradient was repeated twice with orthogonal gradients. Subsequently the ${ }^{13} \mathrm{C}$ spin was excited using a $19.45-\mu \mathrm{s} 90^{\circ}\left({ }^{13} \mathrm{C}\right)$ pulse. This preparation sequence was followed by the pulse sequence of the individual experiments (see Fig. 10). Finally the ${ }^{13} \mathrm{C}$ signal was detected by measuring 512 points during $452 \mathrm{~ms}$. In order to improve the sensitivity, we decoupled all ${ }^{1} \mathrm{H}$ spins during the detection period by applying the DIPSI-2 [22] decoupling sequence.

\section{CONCLUSION}

In this article, we showed how the KL algorithm is a special case of a generalized AJL algorithm. Using the KL algorithm, we obtained a unitary representation of the three-strand braid group and discussed a method for computing the Jones polynomial using this representation over a range of complex numbers. Next, the theory of an idealized NMR quantum computer was presented and we showed how the trace of a unitary matrix can be experimentally determined. Experimental realization for three different knots were performed where the experimental data agreed with theoretical calculations. Future work includes generalizing the AJL algorithm for calculation at a range of values, as was done for the three-strand braid group in this work, and their experimental implementations.

\section{ACKNOWLEDGMENTS}

A.F. thanks NIH GM47467. S.G. acknowledges support from the integrated EU project QAP, the Deutsche Forschungsgemeinschaft (SFB-631), and from the Elite Network of Bavaria program QCCC. Pictures for knots and links were created using KnotPlot, http://knotplot.com.
[1] V. F. R. Jones, Bull. Am. Math. Soc. 12, 103 (1985).

[2] D. Aharonov, V. Jones, and Z. Landau, Algorithmica 55, 395 (2009).

[3] S. J. Lomonaco Jr. and L. H. Kauffman, Proc. SPIE 6244, 62440Z (2006).

[4] L. H. Kauffman and S. J. Lomonaco Jr., in Mathematics of Quantum Computation and Quantum Technology, edited by G. Chen, L. H. Kauffman, and S. J. Lomonaco Jr. (Chapman \& Hall/CRC Pub., 2008), pp. 409.

[5] L. H. Kauffman and S. J. Lomonaco Jr., A 3-Stranded Quantum Algorithm for the Jones Polynomial, Proceeding SPIE, Vol. 6573 (2007), 65730T-1-65730T-13. http://arXiv.org/abs/0706.0020.
[6] D. G. Cory, A. F. Fahmy, and T. F. Havel, in Procedings of the 4th Workshop on Physics and Computation (New England Complex Systems Institute, Boston, MA, 1996), pp. 87.

[7] D. G. Cory, A. F. Fahmy, and T. F. Havel, Proc. Natl. Acad. Sci. USA 94, 1634 (1997).

[8] L. H. Kauffman, Topology 26, 395 (1987).

[9] L. H. Kauffman, Am. Math. Monthly 95, 195 (1988).

[10] L. H. Kauffman, AMS Contemp. Math. Series 78, 263 (1989).

[11] L. H. Kauffman, Temperley-Lieb Recoupling Theory and Invariants of Three-Manifolds (Princeton University Press, Annals Studies, 1994), Vol. 114. 
[12] L. H. Kauffman, Knots and Physics (World Scientific, Singapore, 1991), 2nd ed. (1993); 3rd ed. (2002).

[13] L. H. Kauffman and S. J. Lomonaco, Int. J. Mod. Phys. B 22, 5065 (2008).

[14] S. J. Lomonaco and L. H. Kauffman, Proc. SPIE on Quant. Inform. Comput. VI, 6976, 69760K-1 (2008).

[15] L. H. Kauffman and S. J. Lomonaco Jr., J. Knot Theory and its Ramifications 16, 267 (2007), e-print arXiv:quant-ph/0606114.

[16] P. Shor and S. Jordan, Quantum Inform. Compu. 8, 681 (2008).

[17] J. M. Myers, A. F. Fahmy, S. J. Glaser, and R. Marx, Phys. Rev. A 63, 032302 (2001).
[18] R. R. Ernst, G. Bodenhausen, and A. Wokaun, Principles of Nuclear Magnetic Resonance in One and Two Dimensions (Oxford University Press, Oxford, UK, 1987).

[19] A. F. Fahmy, R. Marx, W. Bermel, and S. J. Glaser, Phys. Rev. A 78, 022317 (2008).

[20] E. Knill and R. Laflamme, Phys. Rev. Lett. 81, 5672 (1998).

[21] A. Barenco, C. H. Bennett, R. Cleve, D. P. DiVincenzo, N. Margolus, P. Shor, T. Sleator, J. Smolin, and H. Weinfurter, Phys. Rev. A 52, 3457 (1995).

[22] A. J. Shaka, C. J. Lee, and A. Pines, J. Magn. Reson. 77, 274 (1988). 